\title{
Doppler Ultrasound Flow Evaluation of the Uterine Arteries Significantly Correlates with Tumor Size in Cervical Cancer Patients
}

\author{
Daniele Bolla, MD ${ }^{1}$, Sarah In-Albon, $\mathrm{MD}^{1}$, Andrea Papadia, MD, $\mathrm{PhD}^{1}$, Edoardo Di Naro, $\mathbf{M D}^{2}$, \\ Maria Luisa Gasparri, MD $^{3}$, Michael M. Mueller, MD $^{1}$, and Luigi Raio, MD ${ }^{1}$ \\ ${ }^{1}$ Department of Gynecology and Obstetrics, University of Bern, Bern, Switzerland; ${ }^{2}$ Department of Gynecology and \\ Obstetrics, University of Bari, Bari, Italy; ${ }^{3}$ Department of Gynecology and Obstetrics, "Sapienza" University of Rome, \\ Rome, Italy
}

\begin{abstract}
Purpose. The aim of this present study was to evaluate the sonographic correlation between Doppler flow characteristics of the uterine arteries and tumor size in patients with cervical cancer, in order to establish a new potential marker to monitor treatment response.
\end{abstract}

Methods. This was a retrospective cohort study of 25 patients who underwent a sonographic evaluation of Doppler flow characteristics of the uterine arteries before surgery or radiochemotherapy for early and locally advanced/advanced cervical cancer, respectively, was analyzed. The primary outcome was the correlation between Doppler flow characteristics of the uterine arteries and tumor size in patients with cervical cancer.

Results. Median age was 49 (range 26-85) years, and mean tumor size was $40.8 \pm 17 \mathrm{~mm}$. A significant positive correlation was found between tumor diameter and the uterine artery end-diastolic velocity $(r=0.47, p<0.05)$ as well as the peak systolic velocity $(r=0.41, p<0.05)$. No correlation was found between tumor size and the pulsatility index or resistance index.

Conclusions. In cervical cancer, uterine artery velocity parameters are associated with tumor size. This finding could become particularly useful in the follow-up of locally advanced cervical cancer patients undergoing radiochemotherapy or in corroborating the selection of

(C) Society of Surgical Oncology 2015

First Received: 21 April 2015;

Published Online: 3 June 2015

A. Papadia, MD, PhD

e-mail: andrea.papadia@insel.ch women with more possibility of a high response rate during neoadjuvant chemotherapy before surgery.

In the last decade, the incidence of mortality due to cervical cancer has markedly decreased in developed countries. Despite this, an estimated 54,000 women are diagnosed with cervical cancer and 25,000 women die from the disease each year in Europe. ${ }^{1}$ Prevention programs, as well as early diagnosis of cervical cancer, play an important role in improving patient survival. Much effort has been undertaken to understand the biological process of this tumor in order to develop new therapeutic targets. Cervical cancer is a highly vascularized tumor that grows 'per continuitatem'. Through angiogenesis, factors such as vascular endothelial growth factors and fibroblast growth factors are able to stimulate an uncoordinated proliferation of vessels. ${ }^{2}$ Transvaginal ultrasonography is now a well-established diagnostic tool for evaluating certain gynecological diseases, with several studies reporting its accuracy for evaluating tumor size and local extension of cervical cancer, showing similar sensitivity/specificity as magnetic resonance imaging (MRI). ${ }^{3,4}$ An accurate stadiation with a focus on extension of cervical cancer is mandatory for the choice of treatment; an early stage could be managed with surgical treatment. On the other hand, concurrent chemoradiation is recognized worldwide as the gold-standard treatment for newly diagnosed, locally advanced cervical cancer (NCI). However, in the last 20 years, neoadjuvant chemotherapy followed by radical surgery, as an alternative to chemoradiation, has been introduced in the management of this malignancy. ${ }^{5}$

Suspicious areas caused by abnormal/irregular vascularization of the cervix can be simply identified via Doppler, and their intratumoral blood flow characteristics 
can be correlated with some tumoral features of the cervical cancer, ${ }^{6}$ such as local extension. However, the correlation between blood flow in the uterine arteries and cervical cancer has been poorly investigated. ${ }^{7}$

The aim of this present study was to evaluate whether there is a sonographic correlation between the Doppler flow characteristics of the uterine arteries and tumor diameter of patients with histologically confirmed cervical cancer.

\section{MATERIALS AND METHODS}

A retrospective single cohort study was conducted between January 2006 and December 2013, including all patients with a diagnosis of cervical cancer who underwent a sonographic Doppler flow examination of the uterine arteries before radiochemotherapy in locally advanced/advanced tumor or surgical treatment alone in the early stages of disease. The ultrasound examinations were performed by two gynecologists with particular ultrasound Doppler study expertise. Inclusion criteria consisted of histologically-confirmed cervical cancer, a Doppler assessment of the uterine arteries prior to any oncologic treatment, and no history of embolization of the uterine arteries. Data were collected from patients' medical records, including age, menopausal status, tumor stage according to the FIGO criteria (International Federation of Gynaecology and Obstetrics staging system), tumor diameter ( $\mathrm{mm}$ ), and Doppler flow characteristics of both uterine arteries [pulsatility index (PI), resistance index (RI), peak systolic velocity (PSV), end-diastolic velocity (EDV)]. To simplify our statistical analysis, the FIGO stages were grouped into four classes: 1 (1A-1B), 2 (2A-2B), 3 (3A-3B), and 4 (4A-4B). A transvaginal ultrasound was performed after diagnosis of cervical cancer using a Voluson 730 (GE Ultrasound, Glattbrugg, Switzerland), a GE E8 (GE Ultrasound), and Acuson Sequoia (Siemens AG, Erlangen, Germany) equipped with a 4-9 MHz endovaginal probe with color and pulsed Doppler capabilities.

Cervical tumoral tissue is usually hypoechogenic due to an impressive intratumural vascularization that can be shown by color or power Doppler sonography. Maximal tumor diameter was measured in millimeters. The uterine artery was identified by color Doppler ultrasonography. The high-pass filter was set at minimum $(60 \mathrm{~Hz})$ and a large sample volume was used for the pulsed Doppler recording to ensure that the blood vessel was uniformly insonated. The angle of insonation was kept below $15^{\circ}$ in all cases, and angle correction was used if the angle was not zero. Three to five uniform waveforms were obtained in succession and online measurements were performed. The PSV, EDV, PI, and RI were calculated for each uterine artery. Mean (left and right uterine artery/2), maximum, and minimum PSV, as well as EDV Doppler flow parameters from both arteries, were calculated and used for statistical purposes. These Doppler characteristics were correlated to the measured tumor size. Data analysis was performed using Prism 5 for Mac OS X, and correlations were searched using the Spearman rank test. Nonparametric variables were analyzed using the Mann-Whitney test, and a $p$ value $<0.05$ was considered significant.

Ethical approval for the current study was obtained by the local Institutional Review Board (Ethics Committee of the Canton of Bern, Switzerland), and the study met the guidelines of the governmental agency responsible.

\section{RESULTS}

A total of 25 patients with histologically-confirmed cervical cancer were included in our study. The median age of the study population was 49 (range 26-85) years, 11 of which were postmenopausal. Twenty-three patients had a squamous cell carcinoma and two had an adenocarcinoma. Mean tumor size was $40.8 \pm 17 \mathrm{~mm}$. Among the 25 patients, 9/25 (36\%) had FIGO stage 1, 10/25 (40 \%) had FIGO stage 2, 5/25 (20\%) had FIGO stage 3, and 1/25 (4\%) had FIGO stage 4 cervical cancer. The sonographic correlations between the Doppler flow parameters of the uterine arteries and the maximum tumor diameter are summarized in Table 1.

Most of the cervical cancers were characterized by a high density of vessels giving the tumor a hypoechogenic appearance at gray-scale transvaginal sonography (Fig. 1a). Moreover, the angioarchitecture of the tumors often showed a more radiate course of the vessels (Fig. 1b). In all cases, both uterine arteries could be visualized. A significant positive correlation was found between the maximum tumor diameter and the $\operatorname{EDV}_{\text {max }}(r=0.47, p<0.05)$ as well as the $\mathrm{PSV}_{\text {min }}(r=0.41, p<0.05)$ of the uterine arteries. No correlation was found between tumor size and PI or RI ( $p=0.94$ and $p=0.38$, respectively). Similarly, correlations were found between the FIGO stages and Doppler flow velocity parameters $\left(\mathrm{PSV}_{\text {mean }}, \mathrm{EDV}_{\text {mean }}\right.$, and $\left.\mathrm{EDV}_{\text {max }}\right)$ $(p<0.05)$, but not with the PI or RI $(p>0.05)$. Furthermore, a significant correlation was found between tumor diameter and FIGO stages $(r=0.53, p<0.01)$.

\section{DISCUSSION}

Ultrasound imaging of cervical cancer is usually presented as a well-localized, hypoechogenic area within the cervix, characterized by a particular dense vascular angioarchitecture (Fig. 1). In recent years, the interest in intratumoral angiogenesis has increased and it has been recognized as an independent prognostic factor for cervical cancer, including tumor growth and risk of metastasis. ${ }^{8-10}$ Tumor angiogenesis can currently only be assessed after 
TABLE 1 Correlations between the Doppler flow parameters of the uterine arteries and the maximum tumor diameter

\begin{tabular}{lcc}
\hline Uterine artery Doppler flow parameters & Result & Correlation with the tumour size \\
\hline RI & $0.86 \pm 0.2$ & $r=0.01, p=0.94$ \\
PI & $1.93 \pm 0.24$ & $r=0.22, p=0.38$ \\
Highest RI & $0.93 \pm 0.41$ & $r=0.06, p=0.77$ \\
Highest PI & $2.3 \pm 0.85$ & $r=0.43, p=0.08$ \\
Lowest RI & $0.77 \pm 0.11$ & $r=0.35, p=0.16$ \\
Lowest PI & $1.8 \pm 0.79$ & $r=0.06, p=0.758$ \\
Peak systolic velocity (PSV) (cm/s) & & $r=0.41, p=0.04$ \\
Highest PSV & $55.9 \pm 27.5$ & $r=0.97, p=0.016$ \\
Lowest PSV & $34.6 \pm 21.5$ & $r=0.23, p=0.25$ \\
End-diastolic velocity (EDV) (cm/s) & & $r .4 \pm 6.8$ \\
Highest EDV & $7.2 \pm 6.2$ & \\
Lowest EDV & & \\
\hline
\end{tabular}

Data are expressed as mean \pm standard deviation

$P I$ pulsatility index, $R I$ resistance index

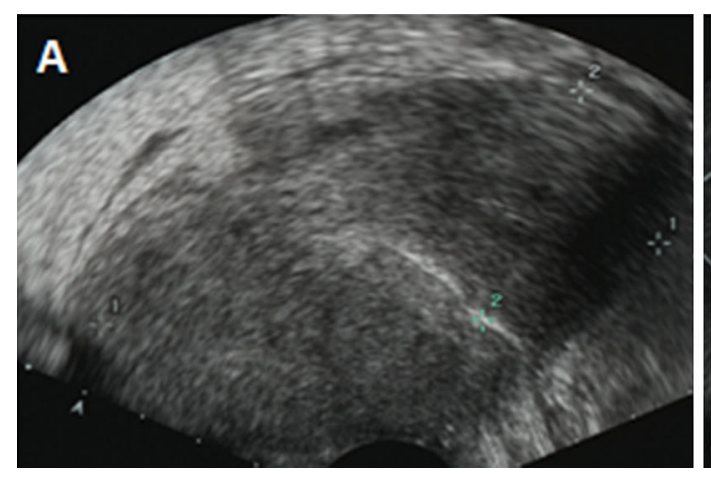

FIG. 1 a Gray-scale transvaginal sonography of a cervical carcinoma showing a hypoecogenic mass within the cervix. b Color Doppler of a horizontal section of the cervix showing a complex

surgery through the measurement of microvessel density within the tumoral tissue. ${ }^{5}$ With the advancement of technical improvements of transvaginal color and pulsed Doppler sonography, the 'in vivo' study of intratumoral blood flow and architecture has became feasible. However, only a few studies have been published to date, and their results were contradictory. ${ }^{11-18}$ Our hypothesis was that the blood flow characteristics in the uterine arteries may be influenced by local expansion of the cervical cancer, increased by neoangiogenesis tumor-linked.

In particular, we showed that the blood flow characteristics of the uterine arteries are influenced by tumor diameter. In fact, the EDV of the uterine arteries was higher with advancing size as well as FIGO stage of the tumor. Moreover, differences in blood flow characteristics of both uterine arteries could only be found by correlating the maximum tumor diameter and the $\mathrm{EDV}_{\max }$ (Fig. 2) as well as the lowest PSV. In our opinion, this can be explained by tumor localization within the cervix which intratumoral angioarchitecture of a cervical cancer. c Color Doppler of a sagittal section of the cervix showing a typical radiate course of the vessels into a cervical cancer

influences the corresponding uterine artery, causing an adaptation of blood flow. Similar uterine artery blood flow behavior can be observed in pregnancy, in which the uterine artery Doppler is considered a valuable tool for the screening of patients at increased risk for pre-eclampsia. ${ }^{19}$ Trophoblastic invasion of the spiral arteries, as well as placental growth, can drammatically influence the blood flow profile of the uterine arteries. However, in pregnancy, with advancing gestational age, the PI and RI decrease. Of interest, local tumor extension and FIGO stage showed a positive correlation with some uterine artery blood flow characteristics; however, the FIGO classification is also influenced by tumor metastasis that can occur independently of tumor size.

To our knowledge, only one previous study evaluated blood flow in the uterine artery in patients with cervical cancer; however, this was carried out for screening purposes and the author concluded that assessment of the uterine artery was not useful due to low sensitivity. ${ }^{13}$ Nevertheless, 


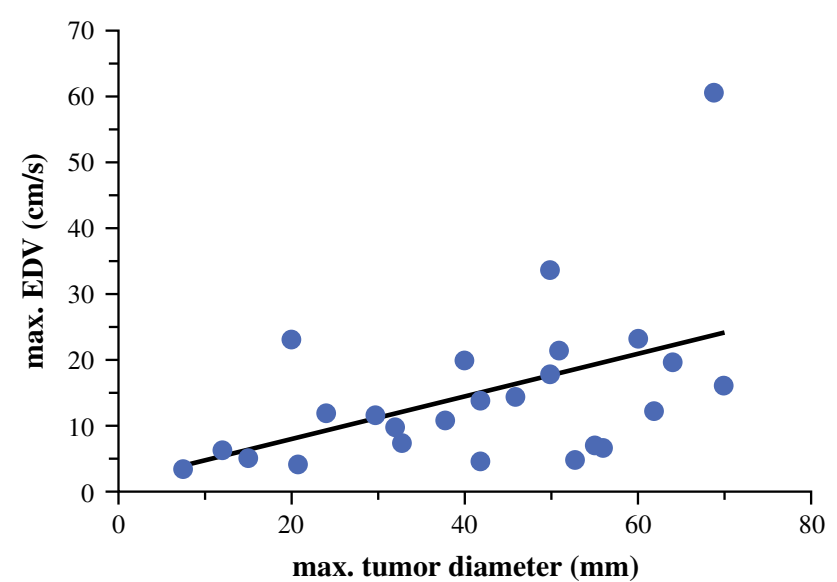

FIG. 2 Correlation between the maximal uterine artery EDV and the maximal tumor diameter $(r=0.47, p<0.05)$. EDV end-diastolic velocity

if our data are confirmed by prospective studies, evaluation of the uterine artery could be particularly useful in the follow-up of patients with cervical cancer during chemotherapy and/or radiotherapy, particularly as it has recently been demonstrated that bevacizumab, an antiangiogenic antibody, increases specific disease-free and overall survival in the advanced stages of gynecological neoplasms ${ }^{20,21}$ including cervical cancer. ${ }^{22}$ Furthermore, a pioneering study hypothesized and assumed that tumor size is strictly correlated to prognosis, ${ }^{5}$ and knowledge of Doppler flow characteristics could be useful in monitoring patients, and could offer speculative advantages in predicting patients who will respond better to treatment. ${ }^{23,24}$ Differences in blood flow characteristics in patients receiving neoadjuvant chemotherapy have also been documented at the time of surgery compared with patients who conducted surgery upfront. $^{25}$

\section{CONCLUSIONS}

Thanks to technical improvements in sonography and, in particular, the transvaginal route, over recent years, investigation of the uterine artery has become simpler and more reproducible. Moreover, in comparison to intratumoral blood flow, a Doppler scan of the uterine arteries may be more objective, and this may help in better understanding the local behavior and biological process of the tumor, favoring more accurate staging and response rate monitoring.

DISCLOSURE Daniele Bolla, Sarah In-Albon, Andrea Papadia, Edoardo Di Naro, Maria Luisa Gasparri, Michael M. Mueller, and Luigi Raio declare they have no commercial interests in the subject of the study. No sources of any financial or material support were used to assist in the preparation of this study.

\section{REFERENCES}

1. WHO/ICO Information Centre on HPV and cervical cancer (HPV Information Centre). Human papillomavirus and related cancers in Europe. Summary report 2010. Barcelona: WHO/ICO HPV Information Centre; 2010.

2. Rasila KK, Burger RA, Smith H, et al. Angiogenesis in gynecological oncology: mechanism of tumor progression and therapeutic targets. Int J Gynecol Cancer. 2005;15:10-726.

3. Byun JM, Kim YN, Jeong DH. Three-dimensional transvaginal ultrasonography for locally advanced cervical cancer. Int $J$ Gynecol Cancer. 2013;23:1459-1464.

4. Testa AC, Ludovisi M, Manfredi R, et al. Transvaginal ultrasonography and magnetic resonance imaging for assessment of presence, size and extent of invasive cervical cancer. Ultrasound Obstet Gynecol. 2009;34:335-344.

5. Benedetti-Panici P, Greggi S, Scambia G, et al. Long-term survival following neoadjuvant chemotherapy and radical surgery in locally advanced cervical cancer. Eur J Cancer. 1998;34:341-6.

6. Alcázar JL, Jurado M, López-García G. Tumor vascularization in cervical cancer by 3-dimensional power Doppler angiography: correlation with tumor characteristics. Int J Gynecol Cancer. 2010;20:393-397.

7. Breyer B, Despot A, Predanic M, et al. Characteristics of blood flow in cancer of the uterine cervix. Ultrasound Obstet Gynecol. 1993;3:268-270.

8. Wiggins D, Granai CO, Steinhoff MM, et al. Tumor angiogenesis as prognostic factor in cervical carcinoma. Gynecol Oncol. 1995;56:353-356

9. Garozzo G, Caragliano L, Consalvo P, et al. Impact of neoangiogenesis on the survival of patients of patients with stage Ib-IIb cervical carcinoma [in Italian]. Minerva Ginecol. 2000;52:73-81.

10. Tjalma W, Van Marck E, Weyler J, et al. Quantification and prognostic relevance of angiogenic parameters in invasive cervical cancer. Br J Cancer. 1998;78:170-174.

11. Cooper RA, Wilks DP, Logue JP, et al. High tumor angiogenesis is associated with poorer survival in carcinoma of the cervix treated with radiotherapy. Clin Cancer Res. 1998;4:2795-2800.

12. Tepper R, Zalel Y, Altaras M, et al. Transvaginal color Doppler ultrasound in the assessment of invasive cervical carcinoma. Gynecol Oncol. 1996;60:26-29.

13. Carter JR. Cervical tumor characterization by transvaginal color flow Doppler ultrasound. Int J Gynecol Cancer. 1999;9:279-284.

14. Hsieh CY, Wu CC, Chen TM, et al. Clinical significance of intratumoral blood flow in cervical carcinoma assessed by color Doppler ultrasound. Cancer. 1995;75:2518-2522.

15. Wu YC, Yuan CC, Hung JH, et al. Power Doppler angiographic appearance and blood flow velocity waveforms in invasive cervical carcinoma. Gynecol Oncol. 2000;79:181-186.

16. Cheng WF, Lee CN, Chu JS, et al. Vascularity index as a novel parameter for the in vivo assessment of angiogenesis in patients with cervical carcinoma. Cancer. 1999;85:651-657.

17. Suren A, Osmers R, Kuhn W. 3D color power angio imaging. A new method to assess intracervical vascularization in benign and pathological conditions. Ultrasound Obstet Gynecol. 1998;11: 133-137.

18. Belitsos P, Papoutsis D, Rodolakis A, et al. Three-dimensional power Doppler ultrasound for the study of cervical cancer and precancerous lesions. Ultrasound Obstet Gynecol. 2012;40(5):576-581.

19. Cnossen JS, Morris RK, Ter Riet G, et al. Use of uterine artery Doppler ultrasonography to predict pre-eclampsia and intrauterine growth restriction: a systematic review and bivariable metaanalysis. CMAJ. 2008;178:701-11. 
20. Bellati F, Napoletano C, Gasparri ML, et al. Current knowledge and open issues regarding bevacizumab in gynaecological neoplasms. Crit Rev Oncol Hematol. 2012;83:35-46.

21. Bellati F, Napoletano C, Gasparri ML, et al. Monoclonal antibodies in gynecological cancer: a critical point of view. Clin Dev Immunol. 2011;2011:890758.

22. Tewari KS, Sill MW, Long HJ 3rd, et al. Improved survival with bevacizumab in advanced cervical cancer. $N$ Engl $\mathrm{J}$ Med. 2014;370:734-43.

23. Greco P, Cormio G, Vimercati A, et al. Transvaginal color Doppler ultrasound for monitoring the response to neoadjuvant chemotherapy in advanced cervical cancer. Acta Obstet Gynecol Scand. 1997;76(2):169-72.

24. Greco P, Cormio G, Vimercati A, et al. Transvaginal color Doppler sonography in predicting the response to chemotherapy in advanced cervical cancer. Ultrasound Obstet Gynecol. 1997; 9:49-52.

25. Bogani G, Cromi A, Serati M, et al. A prospective case-control study on the impact of neoadjuvant chemotherapy on surgeryrelated outcomes of laparoscopic radical hysterectomy. Anticancer Res. 2014;34:5703-8. 